\title{
Tunable nonlinear optical responses based on host- guest MOF hybrid materials
}

\author{
Hongjun $\mathrm{Li}^{\dagger}$, Lin Zhang ${ }^{\dagger}$, Huajun He, Yu Yang, Yuanjing Cui and Guodong Qian
}

\begin{abstract}
The realization of tunable nonlinear optical (NLO) responses in a single nano-/micro-structure is extremely important. However, in lack of effective ways to integrate multiple performances, it still faces severe limitations during applications. Herein, we demonstrate a wavelengthdependent NLO micro-structure based on host-guest metalorganic framework (MOF) materials through encapsulating linear dye molecules into periodic one-dimensional (1D) channels. The confinement to non-centrosymmetric polar dye molecules enhances the second-/third-order NLO responses of the hybrid crystals, causing obvious two-photon luminescence (TPL), second harmonic generation (SHG) and third harmonic generation (THG) responses in the as-prepared composites. The highly ordered structures of MOFs impart spatial regulation on the linear dye molecules to realize orientation alignment, resulting in the polarized anisotropy emission. NIR-to-NIR (NIR, near-infrared region) two-photon pumped lasing is realized with the natural whispering gallery mode resonance cavities of MOFs under the excitation of a 1200-nm fs laser. Furthermore, tunable NLO properties such as TPL, SHG and THG are achieved through switching the incident excitation wavelength from 800 to $1500 \mathrm{~nm}$. Such hybrid materials with tunable NLO responses may open a new avenue toward designing multifunctional NLO devices in the future.
\end{abstract}

Keywords: nonlinear optical response, metal-organic frameworks, second harmonic generation, two-photon luminescence

\section{INTRODUCTION}

Nonlinear optical (NLO) nano-/micro-structure materials are extremely important to materials science communities due to their extensive applications. NLO materials with specific responses are designed and constructed for the targeted application scenarios including information communication, optoelectronics, bio-photonics, and the like [1-3]. Up to now, NLO behaviors especially twophoton luminescence (TPL), second harmonic generation (SHG) and third harmonic generation (THG) have been systematically studied in numerous materials in order to develop various miniature devices [4-6]. Generally, for a certain material, the NLO behavior is specific and unique, which can hardly fulfill the further need for developing multifunctional integrated devices. In this concern, novel materials with multiple NLO properties are highly expected to be explored [7-9]. On the other hand, one single crystal with tunable NLO behaviors seems to be advantageous for integrating and controlling multiple NLO expressions by switching parametric conditions such as excitation wavelength, indicating a simple and effective approach to the aim of multiple NLO properties $[10,11]$. Unfortunately, in lack of appropriate construction strategies to integrate multiple NLO responses in a single device, it is still challenging to compromise structural requirements towards various NLO responses upon one crystal $[12,13]$. It is highly expected that such novel integratable NLO materials with tunable properties, may be beneficial for the future development of optoelectronic devices.

Donor-( $\pi$-spacer)-acceptor (D- $\pi$-A) type organic dyes have been regarded as promising candidates to construct solid-state NLO materials in the fields of multi-photon luminescence and harmonic generation effects [14]. However, the aggregation-caused quenching (ACQ) affects the photoluminescence efficiency of such dyes seriously. Moreover, the organization of dipolar dye molecules in homogeneous orientations is an essential step towards outstanding harmonic performances, espe-

State Key Laboratory of Silicon Materials, Cyrus Tang Center for Sensor Materials and Applications, School of Materials Science and Engineering, Zhejiang University, Hangzhou 310027, China

${ }^{\dagger}$ These two authors contributed equally to this work.

* Corresponding author (email: gdqian@zju.edu.cn) 
cially SHG response. It still suffers from the lack of effective ways to restrict the orientation alignment of $D-\pi$ A molecules [15,16]. Recently, metal-organic frameworks (MOFs) have been intensively researched due to their rational designable structures, flexible functional sites and effective pore confinement [17-19]. Such material could be self-assembled straightforwardly via coordinating metal ions/clusters with organic linkers so that the corresponding structure could be controlled at the molecular level. As novel photonic functional materials, MOFs provide powerful platforms for designing and constructing NLO materials including intrinsic NLO response MOFs and host-guest hybrid NLO MOFs [6,20,21]. MOFs possess unique advantages to construct NLO solidstate microstructures as typical hosts. Firstly, encapsulating organic dyes into MOFs is an effective strategy to homogeneously disperse active chromophores and enhance the optical properties [22,23]. Secondly, smooth surfaces and regular morphology of MOF crystals make them potential natural resonators, providing adequate optical confinement and in turn resulting in enhanced up-conversion luminescence [24,25]. Thirdly, the highly ordered frameworks and designable pores/channels could impart spatial regulation and confinement of guest dipolar molecules, enabling the directional alignment and inducing harmonic generation responses [26]. To the best of our knowledge, although dye-encapsulating MOFs have been utilized to realize one NLO property in a single composite, tunable NLO responses based on MOF hybrid crystals have not been reported yet.

Herein, we demonstrate a hybrid material of LDS722@ZJU-68 with tunable NLO properties based on the host-guest system. The encapsulation of guest dye molecules within porous host MOFs could effectively minimize the ACQ effects and enhance the luminescence properties. NIR-to-NIR (NIR, near infrared region) twophoton pumped lasing (TPPL) could be realized under the excitation of a 1200-nm fs laser from the ZJU-68's natural resonant cavity based on the whispering gallery mode (WGM). The orientation alignment of $\mathrm{D}-\pi-\mathrm{A}$ molecules with the non-centrosymmetric structure along the one-dimensional (1D) channels enhances the second-/third-order NLO responses of the hybrid crystals, causing strong SHG and THG responses. By switching the excitation wavelength from 800 to $1500 \mathrm{~nm}$, we successfully obtained the tunable NLO responses including TPL, TPPL, SHG and THG. Due to the excellent properties of multiple NLO responses, such strategy to construct dye-encapsulating MOF materials might open new routes for the exploration of novel multifunctional NLO devices in the future.

\section{EXPERIMENTAL SECTION}

\section{Materials and general methods}

7-(4-Carboxyphenyl)quinoline-3-carboxylic acid organic linker $\left(\mathrm{H}_{2} \mathrm{CPQC}\right)$ was synthesized according to our previously reported procedures [27]. Organic dye LDS-722 (1-ethyl-4-[4-( $p$-dimethylaminophenyl)-1,3-butadienyl] pyridinium perchlorate) and other reagents of reagent grades were purchased and used without further purification. The powder X-ray diffraction (XRD) data were collected on an X'Pert PRO diffractometer $(\mathrm{Cu} \mathrm{Ka}$ radiation $(\lambda=1.542 \AA)$, room temperature, in the range of $\left.2 \theta=3^{\circ}-50^{\circ}\right)$. The excitation and emission spectra were taken with a Hitachi F-4600 fluorescence spectrometer at room temperature. Thermogravimetric analysis (TGA) was carried out on a Netzsch TG209F3 in $\mathrm{N}_{2}$ atmosphere in the range of $40-800^{\circ} \mathrm{C}$ at the heating speed of $10^{\circ} \mathrm{C} \mathrm{min}{ }^{-1}$. The Fourier transform infrared (FTIR) spectrum was collected on a Thermo Fisher Nicolet iS10 spectrometer in the range of $500-4000 \mathrm{~cm}^{-1}$. For the optical measurements, an optical parametric amplifier (Spirit-OPA + Spirit-OPA-UV3, Newport Corporation) was pumped by a fully automated ultrafast laser system (Spirit One 1040-8, $8 \mathrm{~W}$ at $1040 \mathrm{~nm}$, Newport Corporation), which was used for generating the excitation pulse $(10 \mathrm{kHz}, 800$ to $1500 \mathrm{~nm}$, pulse width < $400 \mathrm{fs})$. The laser beam was focused on the crystals kept on a glass plate, with a $40 \times$ microscope object (numerical aperture $=0.60$ ) in an Olympus IX71 microscope system. The photoluminescence from the sample was collected by the fiber optic spectrometer (PG2000-Pro, Ideaoptics Instruments).

\section{Preparation of ZJU-68}

The synthesis of ZJU-68 was carried out according to our previous work with minor modifications [27]. Zinc tetrafluoroborate hydrate $(40 \mathrm{mg})$ and $\mathrm{H}_{2} \mathrm{CPQC}(20 \mathrm{mg})$ were dissolved in the solution of $\mathrm{N}, \mathrm{N}$-dimethylformamide (DMF, $4 \mathrm{~mL})$, water $(0.1 \mathrm{~mL})$ and acetonitrile $(0.4 \mathrm{~mL})$. The mixture was put in a $20-\mathrm{mL}$ round bottom flask and stirred in an oil bath at $105^{\circ} \mathrm{C}$ for $24 \mathrm{~h}$. Colorless rod-like ZJU-68 crystals were collected by filtration, washed with $\mathrm{DMF}$ and ethanol (EtOH) for several times, and dried in air.

\section{Preparation of LDS-722@ZJU-68}

The method for synthesizing LDS-722@ZJU-68 was similar to that described above, except that LDS-722 dye 
(4 mg) was added into the reaction solution. Purple crystals were filtered, washed with DMF and EtOH for several times in order to remove residual dye from the surface, and then dried in air.

\section{RESULTS AND DISCUSSION}

\section{Synthesis and characterization}

ZJU-68 was selected as the host material in this work and the single crystals were synthesized through a typical solvothermal reaction. The as-synthesized ZJU-68, $\mathrm{H}_{2}\left[\mathrm{Zn}_{3}-\mathrm{O}\left(\mathrm{C}_{17} \mathrm{H}_{9} \mathrm{NO}_{4}\right)_{3}\right]$, exhibit a typical anion framework structure with a class of crystalline porous material with $1 \mathrm{D}$ channels of $6.5 \AA \times 6.5 \AA$ along the $c$-axis as shown in Fig. 1a and Fig. S1. According to our previous work, the $c$-axis direction of ZJU-68 corresponded to the longitudinal direction of the hexagonal crystals, which could be also confirmed by the simulated results of Bravais-Friedel-Donnay-Harker method, as shown in Fig. S2 [27]. ZJU-68 crystallizes in the $P \overline{3}$ space group, which is a typical centrosymmetric structure. In theories, no SHG signals could be observed when excited by an NIR fs laser. Colorless microcrystals display blue light centered at $450 \mathrm{~nm}$ when excited at $365 \mathrm{~nm}$ (Fig. S3). The dipolar linear-shaped laser dye, LDS-722, was selected as guest molecules for its $D-\pi$-A structure which has been extensively researched in NLO fields. Generally, such type of dye molecules possesses typical non-centrosymmetric structures and changeable dipole moments upon excitation, making it possible to realize NLO response when encapsulated into the host matrix [28]. The molecules with a width of approximately $8.0 \AA$ are too large for the ZJU-68 channels so the in situ self-assembly synthetic approach was used as shown in Fig. 1a. Interestingly,
LDS-722@ZJU-68 hybrid crystals were prepared successfully due to the flexibility of organic molecules and tunability of molecular configuration through rotation of bonds. The as-prepared microcrystals exhibit purple appearance (Fig. S3), confirming the successful encapsulation of LDS-722 molecules into the porous MOFs. FTIR spectra (Fig. S4) were performed to further verify the encapsulation. The powder XRD results in Fig. $1 \mathrm{~b}$ indicate that this kind of MOF-based crystalline materials still retain excellent crystallinity and stable framework structure after encapsulating dye molecules into the subnano channels. The as-prepared LDS-722@ZJU-68 crystals exhibit good thermal and chemical stability according to the TGA curves (Fig. S5) and XRD results in different environments (Fig. S6).

\section{Improved optical properties}

The highly ordered structure of ZJU-68 could impart spatial enrichment and confinement of LDS-722 molecules, probably minimizing the ACQ effects and enhancing the optical properties. In order to get insight into the improvement, we examined the emission profiles of LDS722@ZJU-68 hybrid crystals, LDS-722 powder and its DMF solution with the concentration of $1.0 \times 10^{-4} \mathrm{~mol} \mathrm{~L}^{-1}$. Fig. S7 shows the corresponding emission intensity centered at $710 \mathrm{~nm}$ under the excitation of $480 \mathrm{~nm}$. The restricted organic laser dyes in MOFs display strong NIRband emission with quantum yields (QY) of $8.14 \%$, much higher than the QY of LDS-722 powder $(\sim 0.18 \%)$ and LDS-722 solution $(\sim 0.37 \%)$. The corresponding excitation and emission spectra are shown in Fig. S8, and the dye content in the hybrid crystals is calculated to be approximately $4.96 \mathrm{wt} \%$ (Fig. S9). Fig. 2a reveals the corresponding emission spectra at the excitation of $1020 \mathrm{~nm}$,
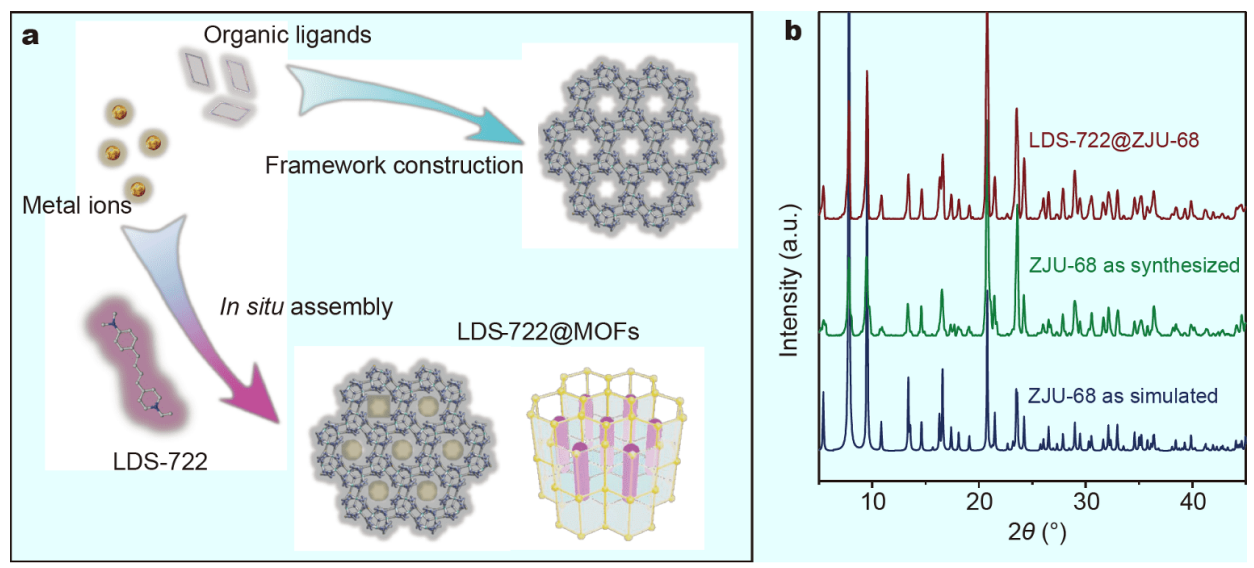

Figure 1 (a) Schematic diagram of synthesizing ZJU-68 and LDS-722@ZJU-68 hybrid microcrystals. (b) The corresponding powder XRD patterns of ZJU-68 and dye-assembled ZJU-68 microcrystals. 

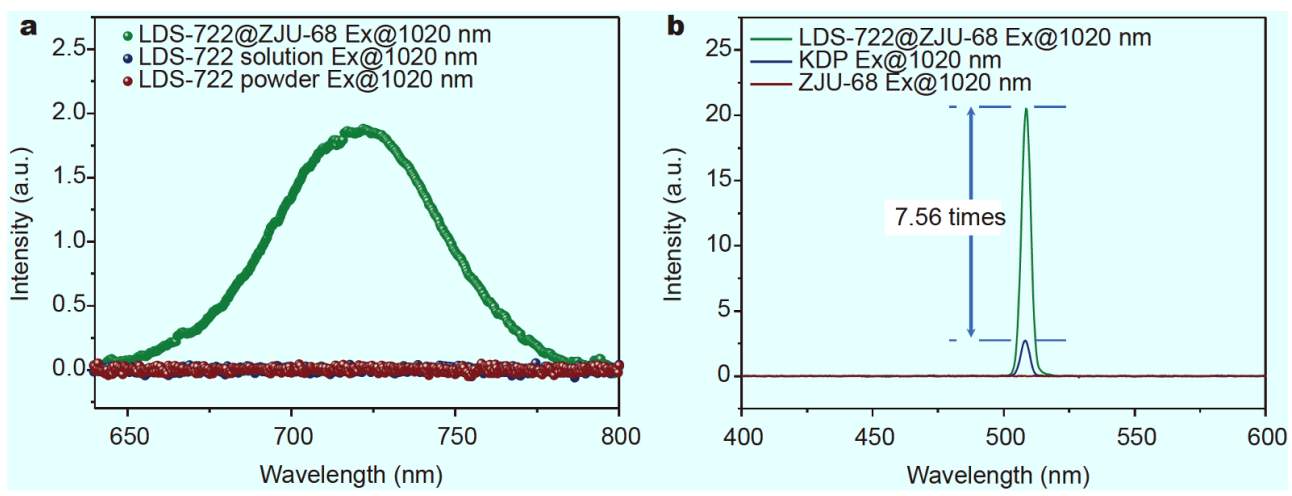

Figure 2 (a) TPL emission spectra of LDS-722 and LDS-722@ZJU-68 excited at 1020 nm. (b) SHG spectra of ZJU-68, KDP, and LDS-722@ZJU-68 crystals excited at $1020 \mathrm{~nm}$.

illustrating that the hybrid crystals exhibit much stronger luminescence than LDS-722 itself. Through changing the excitation energy density, a series of NIR emission peaks centered at $718 \mathrm{~nm}$ could be observed and recorded, as shown in Fig. S10a. Fig. S10b exhibits that the logarithm of the emission intensity at the optimum emission wavelength shows a good linear relationship with the logarithm of the energy density. The resultant slope is approximately $1.91(\approx 2.00)$, indicating the occurrence of TPL emission. The MOF composites exhibit enhanced single-photon and two-photon excited luminescence compared with the original dye powder and solution, proving the significance and necessity of the encapsulation strategy.

Besides, a strong signal at $510 \mathrm{~nm}$ with a sharp peak can be recorded as well (Fig. S10a). The strongest signal wavelength corresponds to half of the excitation wavelength $1020 \mathrm{~nm}$ and Fig. S10c displays the slope of the signal intensity $v s$. excitation energy density on the log-to-log plots of $2.04(\approx 2.00)$, illustrating the occurrence of SHG. For the further investigation of SHG response, we used a common comparison method in the SHG measurement of MOFs and compared the LDS-722, ZJU-68 and LDS722@ZJU-68 crystals with the ground $\mathrm{KH}_{2} \mathrm{PO}_{4}$ (KDP) powders as the reference, which were sieved into the particle size of $25-50 \mu \mathrm{m}$. As shown in Fig. 2b, the SHG efficiency of LDS-722@ZJU-68 is approximately 7.56 times that of KDP powders. No SHG signals could be collected from the excited ZJU-68 crystals as expected. LDS-722 crystals exhibit no obvious SHG response as well due to the strong tendency to align centrosymentrically in dye crystals, leading to the cancellation of individual dipole moments and decrease of the SHG activity [29]. LDS-722@ZJU-68 hybrid crystals exhibit a strong SHG response probably due to the dipolar linear molecules in uniform orientation along the $c$-axis of ZJU-68 crystals. Such orientational arrangement of dye molecules is bound to cause the anisotropy of luminescence, reflecting as the polarized emission possibly.

In order to further investigate the TPL and SHG behaviors, we studied such two NLO responses as function of the polarization of the incident light to clarify the anisotropic effects of the NLO signals under the excitation of polarized $1200 \mathrm{~nm}$ fs laser, resulting in the changing excitation angle $(\theta)$ from $0^{\circ}$ (corresponding to the laser polarization direction parallel to the longitudinal direction of MOFs crystals) to $90^{\circ}$ (corresponding to the laser direction perpendicular to the longitudinal direction) and the like. Fig. S11 displays the scheme of the experimental setup, in which the incident laser is focused on rotating crystals through an objective lens [30]. When the energy density is about $5.22 \mathrm{~mJ} \mathrm{~cm}{ }^{-2}$, obvious SHG signal and TPL could be collected at $\theta=0^{\circ}$, as shown in Fig. 3a. Accompanied by the gradual increasing of the excitation angle, both of them decreases monotonically and hardly any emission could be detected at $\theta=90^{\circ}$. As shown in Fig. 3b, c, both the SHG signal and TPL emission of LDS-722@ZJU-68 single crystal have a similar polarization dependence trend. The polarization ratio of SHG, $\rho=\left(I_{\max }-I_{\min }\right) /\left(I_{\max }+I_{\min }\right)$, is approximately 0.99 and the polarization ratio of TPL is approximately 0.99 as well (limited by the spectral intensity sensitivity of our experimental system). It is worth mentioning that such hybrid microcrystal has excellent polarization ratios, which is higher than most reported values for NLO microcrystals [10]. The polarized TPL emission behavior indicates that the dye absorption transition moments, which are approximately along the molecule axis, are highly aligned along the 1D channels of MOF crystals [31]. Meanwhile, the orienting of polar dye molecules in a 

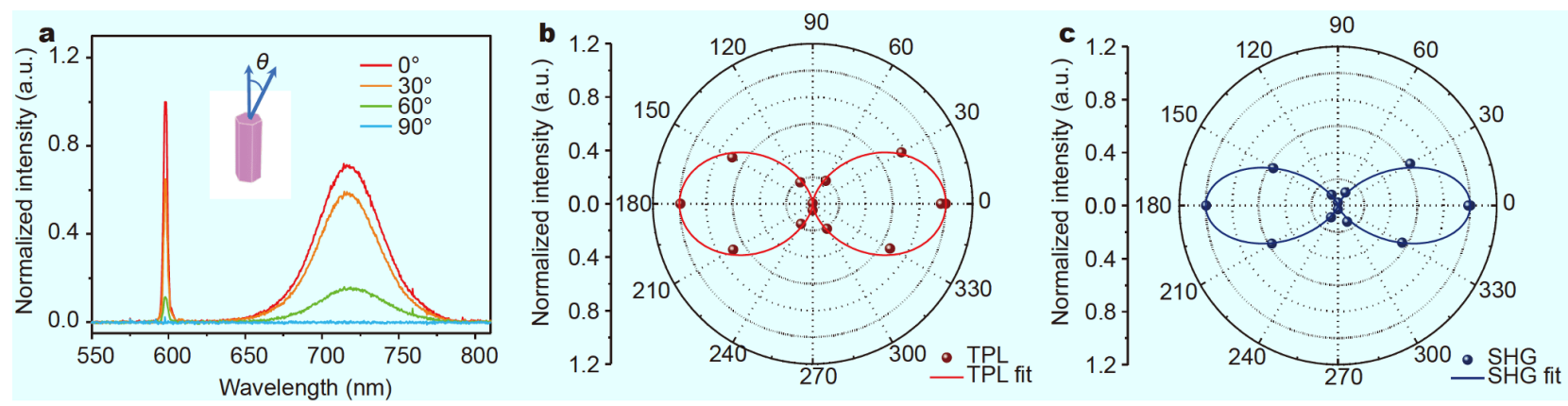

Figure 3 (a) The emission spectra which were analyzed under four different excitation polarizations $\left(\theta=0^{\circ}-90^{\circ}\right)$, excited at $1200 \mathrm{~nm}$. Inset: schematic diagram of the measurement geometry for an individual crystal. (b) TPL and (c) SHG intensity plotted against polarization direction of incident light in LDS-722@ZJU-68 at $1200 \mathrm{~nm}\left(5.22 \mathrm{~mJ} \mathrm{~cm}{ }^{-2}\right)$ from $0^{\circ}$ to $360^{\circ}$. The red and blue lines indicate the $\cos ^{2} \theta$ and $\cos ^{4} \theta$ fits for TPL and SHG, respectively.

preferential order creates a non-centrosymmetric distribution of electric charges, leading to the observation of anisotropic SHG response [28].

\section{Tunable NLO responses}

The strategy of confining organic dye molecules into MOF channels could effectively enhance the optical properties of this hybrid composite to generate not only two-photon excited luminescence, but also effective harmonic generation response. We varied the excitation wavelength of the fs laser from 800 to $1500 \mathrm{~nm}$ for investigating the tunable NLO responses of the hybrid microcrystals. As shown in Fig. $4 \mathrm{a}$, when the $800 \mathrm{~nm}$ laser beam is focused on the hybrid crystal, a bright purple spot can be observed at the excited position. The strongest signal wavelength at $400 \mathrm{~nm}$ corresponds to half of the excitation wavelength, showing a narrow peak with the full wavelength at half maximum (FWHM) of $2.43 \mathrm{~nm}$. The optical signal disappears completely if the excitation position is moved out of the LDS-722@ZJU-68 crystal. A series of emission spectra could be obtained by changing the excitation energy density but no obvious fluorescence could be found. The slope of emission intensity at $400 \mathrm{~nm}$ $v s$. excitation intensity on the log-log plots is $\sim 2.02$ $(\approx 2.00)$, validating the SHG response of LDS-722@ZJU68 composite in the inset of Fig. 4a. Fig. S12a displays the similar experimental phenomenon, showing the strongest SHG signal at $450 \mathrm{~nm}$ with a slope of 1.98 under the excitation of a $900 \mathrm{~nm}$ laser.

When the LDS-722@ZJU-68 microcrystal was excited by $1000 \mathrm{~nm}$ fs laser, the excited position exhibited a broad weak NIR-band fluorescence peak centered at $716 \mathrm{~nm}$, accompanied by an obvious SHG signal at $500 \mathrm{~nm}$ (Fig. 4b). We recorded a series of emission spectra by changing the excitation energy density. The slopes of the NIR fluorescence intensity at the strongest emission wa- velength and SHG intensity as a function of energy density on the log-to-log scale line, both exhibit the second-order dependencies with the slope values of approximately 1.89 and $2.25(\approx 2.00)$, respectively (the insets of Fig. $4 \mathrm{~b}$ ). The microcrystal emits stronger TPL under the excitation of $1100 \mathrm{~nm}$ with an obvious SHG signal at $550 \mathrm{~nm}$ (Fig. S12b). Fig. 4c shows an obvious SHG signal centered at $600 \mathrm{~nm}$ and a single-mode TPPL at around $719 \mathrm{~nm}$ under the excitation of the $1200 \mathrm{~nm}$ fs laser. By varying the energy density, a series of spectra can be recorded. At a low density $\left(<5.32 \mathrm{~mJ} \mathrm{~cm}{ }^{-2}\right)$, the crystals present a broad emission peak centered at $719 \mathrm{~nm}$ with the FWHM of approximately $67 \mathrm{~nm}$, attributed to the spontaneous emission. As the energy density increases, sharp lasing peak at $721 \mathrm{~nm}$ appears abruptly and the output intensity increases rapidly, indicating single-mode lasing operation. The FWHM dropped dramatically down to a much narrow value $(\sim 0.90 \mathrm{~nm})$. Figs S13 and S14 display two bright spots on the excited hybrid crystals, indicating a typical "two-spots" lasing pattern at $1200 \mathrm{~nm}$. The right inset of Fig. 4c shows an obvious knee behavior, which is composed of two fitted linear beelines for the spontaneous emission and lasing region. A relatively low lasing threshold $\left(E_{\mathrm{th}}\right)$ of $\sim 7.74 \mathrm{~mJ} \mathrm{~cm}^{-2}$ is estimated from the intersection of the fitted lines. Such single-mode TPPL is realized by encapsulating the $D-\pi-A$ laser dye molecules into the shortened microcavities, resulting in the achievement of round-trip gain based on WGM according to our previous studies [31,32].

When the excitation wavelength is switched to $1300 \mathrm{~nm}$, NIR fluorescence emission peaks are still observed with a slope of $\sim 1.98(\approx 2.00)$, as shown in Fig. S12c. Apart from TPL emission, there is simultaneous appearance of SHG and THG from the excited crystals when the incident wavelength tunes above $1300 \mathrm{~nm}$. Taking the wavelength at $1400 \mathrm{~nm}$ as an ex- 

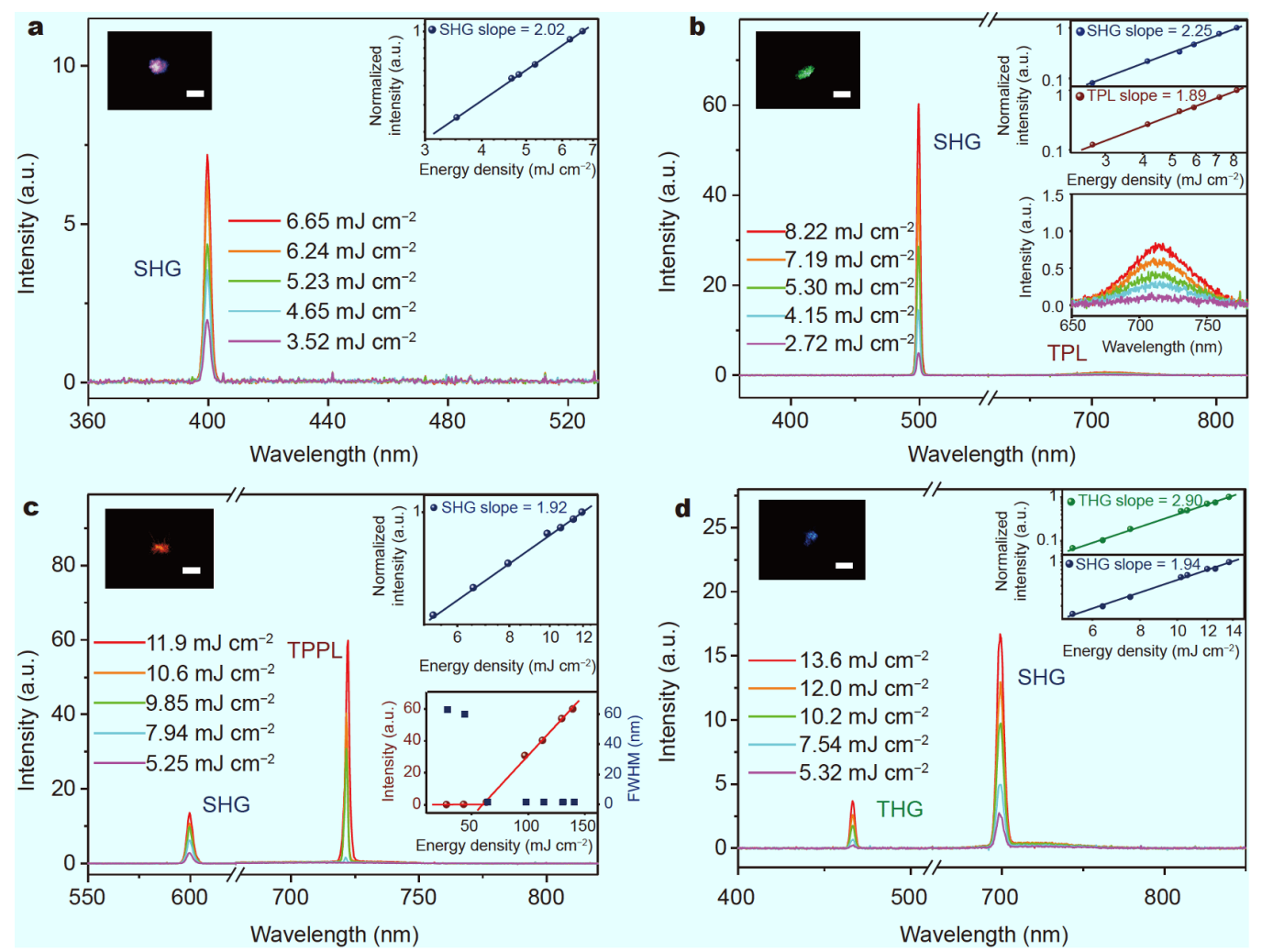

Figure 4 (a) $800 \mathrm{~nm}$ pumped emission spectra of LDS-722@ZJU-68. Insets: the micrograph of LDS-722@ZJU-68 crystal excited at $800 \mathrm{~nm}$ (left) and $\log$-log plots for the SHG intensity $v s$. the excitation energy density, showing a slope of $\sim 2.02$ (right). (b) $1000 \mathrm{~nm}$ pumped emission spectra of LDS722@ZJU-68. Insets: the micrograph of LDS-722@ZJU-68 crystal excited at 1000 nm (left) and log-log plots for the SHG and TPL intensities vs. the excitation energy densities, showing the slopes of 2.25 and 1.89, respectively (right). (c) $1200 \mathrm{~nm}$ pumped emission spectra of LDS-722@ZJU-68. Insets: the micrograph of LDS-722@ZJU-68 crystal excited at $1200 \mathrm{~nm}$ (left) and log-log plots for the SHG intensity vs. the excitation energy density, showing the slope of $\sim 1.92$ (top right) and two-photon emission intensity as a function of pump energy showing the lasing threshold at $\sim 7.74 \mathrm{~mJ} \mathrm{~cm}^{-2}$ and the FWHM showing a dramatical decrease from $\sim 67$ to $\sim 0.9 \mathrm{~nm}$ (bottom right). (d) 1400 nm pumped emission spectra of LDS722@ZJU-68. Insets: the micrograph of LDS-722@ZJU-68 crystal excited at 1400 nm (left) and log-log plots for the SHG and THG intensities vs. the excitation energy densities with the slopes of $\sim 1.94$ and 2.90, respectively (right). Scale bars: $15 \mu \mathrm{m}$.

ample, Fig. 4d shows the obvious SHG signal response at $700 \mathrm{~nm}$ with the slope of approximately $1.94(\approx 2.00)$ for the log-log plots. THG signal can be simultaneously observed, showing the strongest emission wavelength at around $466 \mathrm{~nm}$ corresponding to the one third of the incident wavelength. Basically, SHG intensity scales quadratically with the excitation intensity while that of THG exhibits a cubic dependence on the excitation intensity so that the slope of the strongest signal intensity $v s$. energy density is approximately $2.90(\approx 3.00)$, as shown in the inset of Fig. $4 \mathrm{~d}$. The simultaneous appearance of SHG and THG could be recorded under the excitation of $1500 \mathrm{~nm}$ as well while the TPL is too weak to be observed (Fig. S12d). The obvious THG response is mainly ascribed to specific $D-\pi-A$ dye molecules and the extensive electron coupling within the unique directional dye molecule arrangement in LDS-722@ZJU-68 crystals, boosting the $\chi_{(3)}$ optical nonlinearities and amplifying the third-order NLO property [5]. We studied the THG response of the LDS-722@ZJU-68, ZJU-68 and LDS-722 excited at $1500 \mathrm{~nm}$ and the result is shown in Fig. S15. Through comparing the emission spectra, the hybrid crystals exhibit the stronger THG signal at $500 \mathrm{~nm}$ while the pure ZJU-68 and LDS-722 powders exhibit no obvious THG signal, which further confirms that the THG response is attributed to the introduction of polar molecules.

According to the above results, we summarized the intensity of TPL, SHG and THG signal at approximately the same energy density $\left(\sim 5.50 \mathrm{~mJ} \mathrm{~cm}^{-2}\right)$ from 800 to $1500 \mathrm{~nm}$ in order to obtain the efficient excitation wavelength to generate the tunable NLO properties. Fig. S16 shows the corresponding normalized intensity plots as a function of the excitation wavelength. TPL could be 
realized at a relatively wide region, especially from 1000 to $1300 \mathrm{~nm}$. The largest intensity is obtained under the excitation of $1200 \mathrm{~nm}$, which corresponds to the efficient excitation wavelength of TPL expression. The maximum intensity of SHG is found at excitation wavelengths in the region of around $1000 \mathrm{~nm}$. Meanwhile, we measured the SHG signals from both LDS-722@ZJU-68 and KDP crystals from 800 to $1200 \mathrm{~nm}$ and the details were shown in Figs S17 and S18. By comparing the corresponding intensities, it indicates that such hybrid crystals exhibit excellent SHG response properties at a wide range of excitation wavelength with the SHG efficiency averagely 9 times that of KDP samples. As for THG signal, the block line shows a declining trend from 1500 to $1300 \mathrm{~nm}$, due to parts of the pulsed laser excitation converted to the TPL incoherent processes. According to the above results, we could elaborately select the optimum excitation wavelength to obtain various excellent NLO properties, realizing the tunable NLO properties from a single hybrid material successfully.

\section{CONCLUSIONS}

In summary, for the first time, we have demonstrated the realization of tunable NLO responses, including TPL, SHG and THG in the host-guest hybrid MOF microcrystals through encapsulating the D- $\pi$-A type molecules into the ZJU-68 matrix. The homogeneous separation of LDS-722 molecules in ZJU-68 results in minimized ACQ effects, enhanced optical properties and NIR-to-NIR upconversion lasing. The confinement to non-centrosymmetric polar dye molecules enhances the second-/thirdorder NLO responses of the hybrid crystals, causing obvious TPL, SHG and THG responses in the as-prepared composites. TPPL has been achieved under the excitation of a $1200-\mathrm{nm}$ fs laser with a low threshold of approximately $7.74 \mathrm{~mJ} \mathrm{~cm}^{-2}$. The orientation arrangement of linear dye molecules along the $1 \mathrm{D}$ rigid nanochannels has effectively restricted the rational motion, causing linear alignment of the molecules and resulting in the polarization emission anisotropy. Further, we successfully obtained the tunable NLO responses by switching the excitation wavelength from 800 to $1500 \mathrm{~nm}$, including TPL, SHG and THG. Thus, we have developed a simple but effective strategy to design and construct microstructures with tunable NLO responses, hence offering a useful route to fabricate multifunctional NLO materials in future micro-/nano-photonics.

Received 17 June 2020; accepted 5 July 2020;

published online 14 October 2020
1 Xu J, Li X, Xiong J, et al. Halide perovskites for nonlinear optics. Adv Mater, 2020, 32: 1806736

2 Medishetty R, Zaręba JK, Mayer D, et al. Nonlinear optical properties, upconversion and lasing in metal-organic frameworks. Chem Soc Rev, 2017, 46: 4976-5004

3 Zheng Q, Zhu H, Chen SC, et al. Frequency-upconverted stimulated emission by simultaneous five-photon absorption. Nat Photon, 2013, 7: 234-239

4 Huang J, Guo S, Zhang Z, et al. Designing excellent mid-infrared nonlinear optical materials with fluorooxo-functional group of $\mathrm{d}^{0}$ transition metal oxyfluorides. Sci China Mater, 2019, 62: 17981806

5 Zheng Z, Li D, Liu Z, et al. Aggregation-induced nonlinear optical effects of AIEgen nanocrystals for ultradeep in vivo bioimaging. Adv Mater, 2019, 31: 1904799

6 Aouani H, Rahmani M, Navarro-Cía M, et al. Third-harmonicupconversion enhancement from a single semiconductor nanoparticle coupled to a plasmonic antenna. Nat Nanotech, 2014, 9: 290-294

7 Kharin AY, Lysenko VV, Rogov A, et al. Bi-modal nonlinear optical contrast from Si nanoparticles for cancer theranostics. Adv Opt Mater, 2019, 1801728

8 Mitetelo N, Venkatakrishnarao D, Ravi J, et al. Chirality-controlled multiphoton luminescence and second-harmonic generation from enantiomeric organic micro-optical waveguides. Adv Opt Mater, 2019, 7: 1801775

9 Deska R, Sadecka K, Olesiak-Bańska J, et al. Nonlinear plasmonics in eutectic composites: Second harmonic generation and twophoton luminescence in a volumetric $\mathrm{Bi}_{2} \mathrm{O}_{3}$ - $\mathrm{Ag}$ metamaterial. Appl Phys Lett, 2017, 110: 031102

10 Xiong J, Li X, Yuan C, et al. Wavelength dependent nonlinear optical response of tetraphenylethene aggregation-induced emission luminogens. Mater Chem Front, 2018, 2: 2263-2271

11 Kumar KVA, Rao SV, Hamad S, et al. Wavelength dependent nonlinear optical switching in electron beam irradiated CuTTBPc thin film. RSC Adv, 2016, 6: 22083-22089

12 Venkatakrishnarao D, Narayana YSLV, Mohaiddon MA, et al. Two-photon luminescence and second-harmonic generation in organic nonlinear surface comprised of self-assembled frustum shaped organic microlasers. Adv Mater, 2017, 29: 1605260

13 Su J, Zhang J, Tian X, et al. A series of multifunctional coordination polymers based on terpyridine and zinc halide: Secondharmonic generation and two-photon absorption properties and intracellular imaging. J Mater Chem B, 2017, 5: 5458-5463

14 He GS, Tan LS, Zheng Q, et al. Multiphoton absorbing materials: Molecular designs, characterizations, and applications. Chem Rev, 2008, 108: 1245-1330

15 Kim HS, Lee SM, Ha K, et al. Aligned inclusion of hemicyanine dyes into silica zeolite films for second harmonic generation. J Am Chem Soc, 2004, 126: 673-682

16 Pham TCT, Kim HS, Yoon KB. Large increase in the second-order nonlinear optical activity of a hemicyanine-incorporating zeolite film. Angew Chem Int Ed, 2013, 52: 5539-5543

17 Zhao D, Timmons DJ, Yuan D, et al. Tuning the topology and functionality of metal-organic frameworks by ligand design. Acc Chem Res, 2011, 44: 123-133

18 Jiao L, Wang Y, Jiang HL, et al. Metal-organic frameworks as platforms for catalytic applications. Adv Mater, 2018, 30: 1703663

19 Niu Z, Cui X, Pham T, et al. A metal-organic framework based 
methane nano-trap for the capture of coal-mine methane. Angew Chem Int Ed, 2019, 58: 10138-10141

20 Nguyen TN, Ebrahim FM, Stylianou KC. Photoluminescent, upconversion luminescent and nonlinear optical metal-organic frameworks: From fundamental photophysics to potential applications. Coord Chem Rev, 2018, 377: 259-306

21 Cui Y, Zhang J, He H, et al. Photonic functional metal-organic frameworks. Chem Soc Rev, 2018, 47: 5740-5785

$22 \mathrm{Li} \mathrm{H}, \mathrm{He} \mathrm{H}, \mathrm{Yu}$ J, et al. Dual-band simultaneous lasing in MOFs single crystals with Fabry-Perot microcavities. Sci China Chem, 2019, 62: 987-993

23 Wei Y, Dong H, Wei C, et al. Wavelength-tunable microlasers based on the encapsulation of organic dye in metal-organic frameworks. Adv Mater, 2016, 28: 7424-7429

$24 \mathrm{Yu}$ J, Cui Y, Xu H, et al. Confinement of pyridinium hemicyanine dye within an anionic metal-organic framework for two-photonpumped lasing. Nat Commun, 2013, 4: 2719

25 Venkatakrishnarao D, Mamonov EA, Murzina TV, et al. Advanced organic and polymer whispering-gallery-mode microresonators for enhanced nonlinear optical light. Adv Opt Mater, 2018, 6: 1800343

26 Yu J, Cui Y, Wu C, et al. Second-order nonlinear optical activity induced by ordered dipolar chromophores confined in the pores of an anionic metal-organic framework. Angew Chem Int Ed, 2012, 51: $10542-10545$

27 He H, Ma E, Cui Y, et al. Polarized three-photon-pumped laser in a single MOF microcrystal. Nat Commun, 2016, 7: 11087

28 Sola-Llano R, Martínez-Martínez V, Fujita Y, et al. Formation of a nonlinear optical host-guest hybrid material by tight confinement of LDS 722 into aluminophosphate 1D nanochannels. Chem Eur J, 2016, 22: 15700-15711

29 Kim HS, Pham TT, Yoon KB. Aligned inclusion of dipolar dyes into zeolite channels by inclusion in the excited state. J Am Chem Soc, 2008, 130: 2134-2135

30 He H, Zhang X, Yan X, et al. Broadband second harmonic generation in GaAs nanowires by femtosecond laser sources. Appl Phys Lett, 2013, 103: 143110

$31 \mathrm{He} \mathrm{H}, \mathrm{Ma} \mathrm{E}, \mathrm{Yu}$ J, et al. Periodically aligned dye molecules integrated in a single MOF microcrystal exhibit single-mode linearly polarized lasing. Adv Opt Mater, 2017, 5: 1601040

32 Tang B, Sun L, Zheng W, et al. Ultrahigh quality upconverted single-mode lasing in cesium lead bromide spherical microcavity. Adv Opt Mater, 2018, 6: 1800391

Acknowledgements This work was supported by the National Natural Science Foundation of China (51632008, U1609219 and 61721005).

Author contributions $\quad \mathrm{Li} \mathrm{H}$ and Zhang $\mathrm{L}$ designed and performed the experiments, analyzed the data and wrote the paper; He H, Yang $\mathrm{Y}$ and Cui Y analyzed the data; Qian G conceived the framework of this paper and revised the paper. All authors contributed to the general discussion.

Conflict of interest The authors declare that they have no conflict of interest.

Supplementary information online version of the paper.

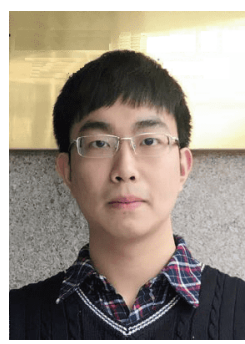

Hongjun Li received his Bachelor's degree in materials science and engineering from Northeastern University (2017). He is currently pursuing a PhD at the School of Materials Science and Engineering at Zhejiang University under the supervision of Prof. Guodong Qian. His research interest focuses on photonic MOF materials.

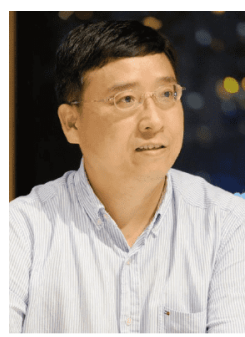

Guodong Qian received his Bachelor's (1988) and Master's (1992) degrees in materials science from Zhejiang University. He was promoted to Associate Professor, Full Professor, and Cheung Kong Professor in 1999, 2002, and 2011, respectively. His current research interests include hybrid organic-inorganic photonic functional materials and multifunctional porous materials.

\section{基于主-客体型金属-有机框架材料的可调谐非线} 性光学性能

\author{
李鸿钧 ${ }^{\dagger}$, 张琳 $^{\dagger}$, 何桦浚, 杨雨, 崔元靖, 钱国栋 ${ }^{*}$
}

摘要 本文通过采用原位自组装工艺将线形有机染料分子组装在 具有一维孔道的金属-有机框架材料(MOFs)中, 成功制备了基于 主-客体系的复合晶态材料. MOFs的高度有序微孔结构赋予线形 染料分子以强烈的限域作用, 促使染料分子一维定向排列, 从而实 现材料的光学性能各向异性. 相比于无限域染料分子, 该复合材料 展示出显著增强的双光子发光性能(TPL), 并利用MOFs单晶晶面 形成的天然谐振腔实现了近红外波段的双光子激光(TPPL). MOFs 孔道中极性染料分子的有序组装使该复合晶体产生二阶/三阶非线 性光学响应, 获得了强的二次谐波 (SHG)和三次谐波 (THG)效应. 在800-1500 nm范围内调谐激发波长, 该材料展示出激发波长依赖 的非线性光学响应调谐性能, 获得TPL、TPPL、SHG和THG的多 重非线性光学性能. 上述结果为未来设计高效的非线性光学器件 开辟了一条简单有效的途径. 